\title{
Symmetric Radial Basis Function Assisted Space-Time Equalisation for Multiple Receive-Antenna Aided Systems
}

\author{
S. Chen, S. Liu and L. Hanzo \\ School of ECS, \\ University of Southampton, SO17 1BJ, UK \\ www-mobile.ecs.soton.ac.uk; lh@ecs.soton.ac.uk
}

\begin{abstract}
This constribution considers nonlinear space-time equalisation (STE) designed for single-input multiple-output (SIMO) systems. By exploiting the inherent symmetry of the underlying optimal Bayesian STE solution, a novel symmetric radial basis function (RBF) based STE scheme is proposed, which is capable of achieving the optimal Bayesian equalisation performance. The adaptive adjustment of the STE taps of this symmetric RBF (SRBF) based STE can be achieved by estimating the SIMO channel encountered using the classic least mean square channel estimator and computing the optimal RBF centres from the resultant SIMO channel matrix estimate. Our simulation results demonstrate that the performance of this SRBF based STE is robust with respect to the choice of the algorithmic parameters.
\end{abstract}

\section{INTRODUCTION}

Space-time processing techniques play an increasingly important role in wireless communications [1]-[7]. With the aid of smart antenna arrays and by exploiting both the space and time dimensions, space-time processing becomes capable of improving the achievable system capacity, coverage and quality of service by suppressing both the intersymbol interference and the co-channel interference. The family of singleinput multiple-output (SIMO) systems has enjoyed popularity owing to its potent performance benefits and relative implementational simplicity. A SIMO system consists of a singleantenna transmitter and a receiver equipped with multiple antennas. A space-time equaliser (STE) [8]-[12] based on this SIMO structure is capable of mitigating the channel

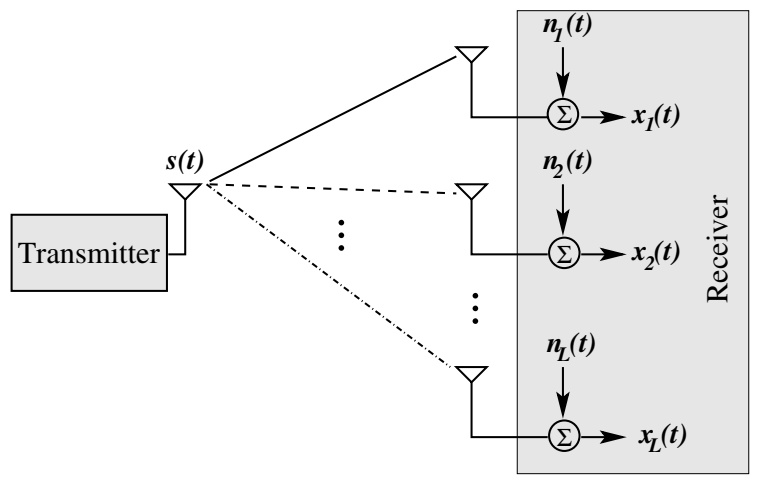

Fig. 1. Single-input multiple-output system employing multiple receive antennas. impairments arising from hostile multipath propagation. The most commonly used STE structure is linear and classically the design of such a linear STE is based on the minimum mean square criterion. A recent study has shown that the optimal linear STE design - optimal in terms of attaining the Minimum Bit Error Ratio (MBER) - is the one that adopts the minimum bit error rate criterion of [12], and hence we refer to this STE design as the linear MBER STE.

However, we will show that the true optimal STE is nonlinear, just as in the case of single-input single-output (SISO) systems [13]-[16]. We will highlight the inherent symmetry of the optimal Bayesian STE solution's modulated signal constellation, and demonstrate that this enables us to propose an adaptive nonlinear STE based on a novel symmetric radial basis function (RBF) network. A convenient way of adaptively adjusting the taps of this symmetric RBF (SRBF) STE is to estimate the SIMO channels using the classic least mean square (LMS) channel estimator and then to compute the optimal RBF centre vectors based on the resultant SIMO channel matrix estimate. This adaptive SRBF STE is capable of realising the optimal Bayesian STE and its performance is robust with respect to the choice of the algorithmic parameters, such as the RBF centres' variance value, as will be demonstrated with the aid of our simulations.

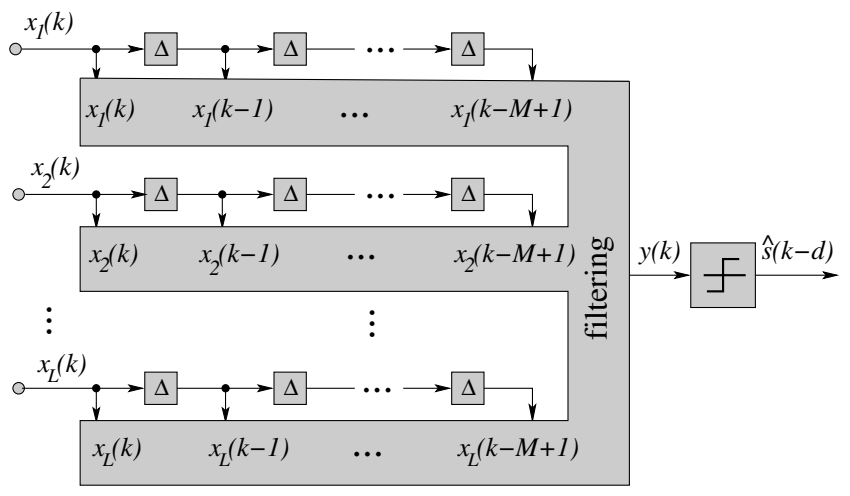

Fig. 2. Space-time equalisation structure using $\Delta$-spaced temporal filters, where $\Delta=T_{s}$ and $T_{s}$ denotes the symbol period, $M$ is the temporal filter order, and $d$ the decision delay. 


\section{SIMO SYSTEM MODEL}

A SIMO system employs a single transmit antenna and $L(>$ 1) receive antennas, as depicted in Fig. 1, where $s(t)$ is the transmitted signal, $x_{l}(t)$ denotes the $l$-th receiver antenna's output signal and $n_{l}(t)$ the $l$-th channel's noise. The received signals are sampled at the symbol rate to obtain the $L$ antennas' output samples $x_{l}(k), 1 \leq l \leq L$, which are passed to a generic STE, as shown in Fig. 2. The received signal sample $x_{l}(k)$ of the $l$-th antenna can be expressed as

$$
x_{l}(k)=\sum_{i=0}^{n_{c}-1} c_{i, l} s(k-i)+n_{l}(k)=\bar{x}_{l}(k)+n_{l}(k),
$$

where $n_{l}(k)$ is a complex-valued Gaussian white noise process with $E\left[\left|n_{l}(k)\right|^{2}\right]=2 \sigma_{n}^{2}, \bar{x}_{l}(k)$ denoting the noise-free part of the $l$-th channel's output. The modulation scheme is assumed to be binary phase shift keying (BPSK) and therefore the transmitted symbol sequence $s(k)$ assumes values from the BPSK symbol set $\{ \pm 1\}$, while $c_{i, l}$ represents the complexvalued taps of the $l$-th channel impulse response (CIR) having a length of $n_{c}$ taps. For notational simplicity, we have assumed that each of the $L$ SIMO channels has the same length of $n_{c}$. The task of the STE is to use the multi-channel output vector

$$
\mathbf{x}(k)=\left[\mathbf{x}_{1}^{T}(k) \mathbf{x}_{2}^{T}(k) \cdots \mathbf{x}_{L}^{T}(k)\right]^{T}
$$

to produce an estimate $\hat{s}(k-d)$ of the transmitted symbol $s(k-d)$, where $d$ denotes the STE's decision delay,

$$
\mathbf{x}_{l}(k)=\left[x_{l}(k) x_{l}(k-1) \cdots x_{l}(k-M-1)\right]^{T}
$$

for $1 \leq l \leq L$, and $M$ is the order of the $l$-th temporal filter. Again, for notational simplicity, we have assumed that each of the $L$ temporal filters of the STE has the same order $M$. In most state-of-the-art applications a linear - rather than nonlinear - STE has been employed, whose soft output is given by

$$
y(k)=\sum_{l=1}^{L} \sum_{i=0}^{M-1} \theta_{i, l}^{*} x_{l}(k-i)=\boldsymbol{\theta}^{H} \mathbf{x}(k),
$$

where $\boldsymbol{\theta}=\left[\begin{array}{lll}\boldsymbol{\theta}_{1}^{T} & \boldsymbol{\theta}_{2}^{T} \cdots \boldsymbol{\theta}_{L}^{T}\end{array}\right]^{T}$, and $\boldsymbol{\theta}_{l}=\left[\begin{array}{lll}\theta_{0, l} & \theta_{1, l} \cdots \theta_{M-1, l}\end{array}\right]^{T}$ is the $l$-th temporal filter's weight vector. Again, the optimal solution for the linear STE's weight vector $\boldsymbol{\theta}$ is known to be the linear minimum bit error rate (LMBER) solution [17]. However, just as in the case of the SISO channel equalisation [13]-[16], the optimal STE solution based on the information provided in $\mathbf{x}(k)$ is nonlinear.

\section{BAyesian Space-Time EQUALiser Solution}

Note that the STE's input vector $\mathbf{x}(k)$ can be expressed as

$$
\mathbf{x}(k)=\mathbf{C s}(k)+\mathbf{n}(k)=\overline{\mathbf{x}}(k)+\mathbf{n}(k)
$$

where $\mathbf{n}(k)=\left[\begin{array}{ll}\mathbf{n}_{1}^{T}(k) & \mathbf{n}_{2}^{T}(k) \cdots \mathbf{n}_{L}^{T}(k)\end{array}\right]^{T}$ with $\mathbf{n}_{l}(k)=$ $\left[n_{l}(k) n_{l}(k-1) \cdots n_{l}(k-M+1)\right]^{T}$, the symbol vector

$$
\mathbf{s}(k)=\left[s(k) s(k-1) \cdots s\left(k-M-n_{c}+2\right)\right]^{T},
$$

and the overall CIR matrix $\mathbf{C}$ is defined as

$$
\mathbf{C}=\left[\begin{array}{c}
\mathbf{C}_{1} \\
\mathbf{C}_{2} \\
\vdots \\
\mathbf{C}_{L}
\end{array}\right]
$$

with the $M \times\left(M+n_{c}-1\right)$ CIR matrix $\mathbf{C}_{l}$ given by

$$
\mathbf{C}_{l}=\left[\begin{array}{ccccccc}
c_{0, l} & c_{1, l} & \cdots & c_{n_{c}-1, l} & 0 & \cdots & 0 \\
0 & c_{0,1} & c_{1, l} & \ldots & c_{c_{n}-1, l} & \ddots & \vdots \\
\vdots & \ddots & \ddots & \ddots & \ldots & \ddots & 0 \\
0 & \cdots & 0 & c_{0,1} & c_{1, l} & \cdots & c_{n_{c}-1, l}
\end{array}\right] .
$$

We define the overall signal to noise ratio (SNR) of the SIMO system under consideration as

$$
\mathrm{SNR}=\frac{\sigma_{s}^{2}}{L 2 \sigma_{n}^{2}} \sum_{l=1}^{L} \sum_{i=0}^{n_{c}-1}\left|c_{i, l}\right|^{2},
$$

where $\sigma_{s}^{2}=1$ is the BPSK symbol energy.

Denote the $N_{s}=2^{M+n_{c}-1}$ legitimate combinations of $\mathbf{s}(k)$ as $\mathbf{s}_{q}, 1 \leq q \leq N_{s}$. Denote furthermore the $d$-th element of $\mathbf{s}_{q}$, corresponding to the desired symbol $s(k-d)$ as $s_{q, d}$. The noiseless SIMO channel output $\overline{\mathbf{x}}(k)$ only takes values from the finite signal state set

$$
\overline{\mathbf{x}}(k) \in \mathcal{X} \triangleq\left\{\overline{\mathbf{x}}_{q}=\mathbf{C s}_{q}, 1 \leq q \leq N_{s}\right\},
$$

which can be divided into two subsets conditioned on the value of $s(k-d)$ as

$$
\mathcal{X}^{( \pm)} \triangleq\left\{\overline{\mathbf{x}}_{q} \in \mathcal{X}, 1 \leq i \leq N_{s b}: s(k-d)= \pm 1\right\},
$$

where the size of $\mathcal{X}^{(+)}$and $\mathcal{X}^{(-)}$is $N_{s b}=N_{s} / 2$. Let the conditional probabilities of receiving $\mathbf{x}(k)$ given $s(k-d)=$ \pm 1 be $p_{ \pm}(\mathbf{x}(k))=p(\mathbf{x}(k) \mid s(k-d)= \pm 1)$. According to the classic Bayesian decision theory [18], the optimal detection strategy is

$$
\hat{s}(k-d)=\left\{\begin{array}{lll}
+1, & \text { if } & p_{+}(\mathbf{x}(k)) \geq p_{-}(\mathbf{x}(k)), \\
-1, & \text { if } p_{+}(\mathbf{x}(k))<p_{-}(\mathbf{x}(k)) .
\end{array}\right.
$$

Let us now introduce the following real-valued Bayesian decision variable

$$
y_{\text {Bay }}(k)=f_{\text {Bay }}(\mathbf{x}(k)) \triangleq \frac{1}{2} p_{+}(\mathbf{x}(k))-\frac{1}{2} p_{-}(\mathbf{x}(k)) .
$$

The optimal detection rule (12) is equivalent to

$$
\hat{s}(k-d)=\operatorname{sgn}\left(y_{\text {Bay }}(k)\right)= \begin{cases}+1, & y_{\text {Bay }}(k) \geq 0, \\ -1, & y_{\text {Bay }}(k)<0 .\end{cases}
$$

Given the signal model of (5), the decision variable (13) can be expressed as

$$
y_{\text {Bay }}(k)=\sum_{q=1}^{N_{s}} \operatorname{sgn}\left(s_{q, d}\right) \beta_{q} e^{-\frac{\left\|\mathbf{x}(k)-\overline{\mathbf{x}}_{q}\right\|^{2}}{2 \sigma_{n}^{2}}}
$$

where $\beta_{q}$ denotes the a priori probability of $\overline{\mathbf{x}}_{q}$. Since all the $\overline{\mathbf{x}}_{q}$ values are equiprobable, all the $\beta_{q}$ values are equal.

Let us now highlight the symmetry of the Bayesian solution. Lemma 1: The two subsets $\mathcal{X}^{(+)}$and $\mathcal{X}^{(-)}$are distributed symmetrically, hence for any signal state $\overline{\mathbf{x}}_{i}^{(+)} \in \mathcal{X}^{(+)}$there exists a signal state $\overline{\mathbf{x}}_{q}^{(-)} \in \mathcal{X}^{(-)}$satisfying $\overline{\mathbf{x}}_{q}^{(-)}=-\overline{\mathbf{x}}_{i}^{(+)}$. Proof: Let $\overline{\mathbf{x}}_{i}^{(+)}=\mathbf{C s}_{i}^{(+)} \in \mathcal{X}^{(+)}$. Then

$$
-\overline{\mathbf{x}}_{i}^{(+)}=\mathbf{C}\left(-\mathbf{s}_{i}^{(+)}\right) \in \mathcal{X}^{(-)} .
$$


Given this symmetry, the optimal Bayesian equalisation solution (15) can be rewritten as

$$
y_{\text {Bay }}(k)=\sum_{q=1}^{N_{s b}} \beta_{q}\left(e^{-\frac{\left\|\mathbf{x}(k)-\overline{\mathbf{x}}_{q}^{(+)}\right\|^{2}}{2 \sigma_{n}^{2}}}-e^{-\frac{\left\|\mathbf{x}(k)+\overline{\mathbf{x}}_{q}^{(+)}\right\|^{2}}{2 \sigma_{n}^{2}}}\right) \text {, }
$$

where $\overline{\mathbf{x}}_{q}^{(+)} \in \mathcal{X}^{(+)}$. The Bayesian STE solution has odd symmetry, as $f_{\text {Bay }}(-\mathbf{x}(k))=-f_{\text {Bay }}(\mathbf{x}(k))$. In the next section, we propose a RBF network which has the same oddsymmetric structure.

\section{Symmetric RBF Space-Time Equaliser Design}

The proposed SRBF network assumes the following form

$$
y_{\mathrm{SRBF}}(k)=f_{\mathrm{SRBF}}(\mathbf{x}(k))=\sum_{i=1}^{N_{s b}} \theta_{i} \phi_{i}(\mathbf{x}(k))
$$

where $f_{\mathrm{SRBF}}(\bullet)$ is a real-valued nonlinear mapping realised by the SRBF network, $\theta_{i}$ is the $i$-th real-valued RBF weight, $\phi_{i}(\bullet)$ denotes the $i$-th RBF unit, and $N_{s b}$ is the number of RBF units used. The SRBF STE makes its decisions according to $\hat{s}(k-d)=\operatorname{sgn}\left(y_{\mathrm{SRBF}}(k)\right)$. In contrast to the standard RBF model, here, we propose to adopt the following symmetric RBF unit

$$
\phi_{i}(\mathbf{x}) \triangleq \varphi\left(\mathbf{x} ; \mathbf{c}_{i}, \sigma_{i}^{2}\right)-\varphi\left(\mathbf{x} ;-\mathbf{c}_{i}, \sigma_{i}^{2}\right),
$$

where $\mathbf{c}_{i} \in \mathcal{C}^{L M}$ is the $i$-th complex-valued RBF centre, $\sigma_{i}^{2}$ the $i$-th real-valued RBF centres' variance, and $\varphi(\bullet)$ the classic RBF function. In this study we adopt the Gaussian RBF function of the form

$$
\varphi\left(\mathbf{x} ; \mathbf{c}_{i}, \sigma^{2}\right)=e^{-\frac{\left\|\mathbf{x}-\mathbf{c}_{i}\right\|^{2}}{2 \sigma^{2}}} .
$$

The SRBF network (17) with the node structure (18) has an inherently odd symmetry, just as the Bayesian STE solution. Its is worth noting that the standard RBF model, where the $\mathrm{RBF}$ node is defined by $\phi_{i}(\mathbf{x}) \triangleq \varphi\left(\mathbf{x} ; \mathbf{c}_{i}, \sigma_{i}^{2}\right)$, is unable to guarantee this odd symmetry.

It is plausible that we can set all the RBF weights according to $\theta_{i}=\theta>0$ and all the RBF centres' variances to $\sigma_{i}^{2}=\hat{\sigma}_{n}^{2}$, where $\hat{\sigma}_{n}^{2}$ is the estimate of $\sigma_{n}^{2}$. Furthermore, if we use the set of signal states $\mathcal{X}^{(+)}$as the RBF centres of this SRBF STE, then the proposed solution becomes capable of exactly achieving the optimal Bayesian performance. A simple and effective means of realising this optimal RBF centre set is to estimate the SIMO CIRs using the LMS algorithm as follows

$$
\hat{c}_{i, l}(k)=\hat{c}_{i, l}(k-1)+\mu_{c} \epsilon_{l}(k) s(k-i), 0 \leq i \leq n_{c}-1,
$$

TABLE I

THE CIRS OF THE SIMULATED SIMO SYSTEM WITH $L=4$ AND $n_{c}=3$. THE ACTUALLY SIMULATED CHANNEL WAS NORMALISED ACCORDING TO

$$
\mathbf{c}_{l} /\left\|\mathbf{c}_{l}\right\| \text {. }
$$

\begin{tabular}{|r|rrr|}
\hline$l$ & \multicolumn{3}{|c|}{$\mathbf{c}_{l}$} \\
\hline 1 & $-0.2+j 0.3$ & $-0.5+j 0.4$ & $0.7-j 0.6$ \\
2 & $-0.3+j 0.2$ & $0.7-j 0.1$ & $-0.5+j 0.4$ \\
3 & $-0.1-j 0.2$ & $0.6+j 0.1$ & $-0.4+j 0.3$ \\
4 & $0.1+j 0.1$ & $0.2+j 0.2$ & $-0.4-j 0.4$ \\
\hline
\end{tabular}

for $1 \leq l \leq L$, where $\mu_{c}$ is the step size, and

$$
\epsilon_{l}(k)=x_{l}(k)-\sum_{i=0}^{n_{c}-1} \hat{c}_{i, l}(k-1) s(k-i) .
$$

From the estimated SIMO channel matrix $\hat{\mathbf{C}}$, it is straightforward to calculate the optimal RBF centre set, namely, $\mathcal{X}^{(+)}$. The estimated noise variance $\hat{\sigma}_{n}^{2}$ is used as the RBF centres' variance. We point out that our experience indicates that the performance of the SRBF STE is not overly sensitive to the value of the RBF centres' variance used, and hence there exists a large range of the RBF centres' variance values which enable the SRBF STE to achieve the optimal Bayesian performance. This will be more explicitly demonstrated in our simulation study. This underlying robustness to the value of the RBF centres' variance is a consequence of the Bayesian detector's robustness to the noise variance $\sigma_{n}^{2}$ used. It has been shown [16] that the performance of the Bayesian detectors using $0.2 \sigma_{n}^{2}$ and $5 \sigma_{n}^{2}$ to substitute the noise variance $\sigma_{n}^{2}$ is indistinguishable from that of the exact Bayesian solution.

\section{Simulation Study}

A SIMO system using $L=4$ receive antennas was simulated, and each channel had $n_{c}=3$ taps. Table I lists the CIRs $\mathbf{c}_{l}^{T}=\left[\begin{array}{lll}c_{0, l} & c_{1, l} & c_{2, l}\end{array}\right], 1 \leq l \leq 4$, of this SIMO system. The simulated channel was normalised according to $\mathbf{c}_{l} /\left\|\mathbf{c}_{l}\right\|$ for the sake of maintaining a unity channel gain. The STE's temporal filter order was chosen as $M=5$. For this example, the STE's decision delay $0 \leq d \leq 6$, and the optimal decision delay was found to be $d=4$. This optimal decision delay was used in our simulations. Fig. 3 depicts the bit error ratio (BER) performance of the two benchmarkers, namely that of the linear MBER STE and of the optimal Bayesian STE.

The proposed SRBF based STE was next investigated. The LMS channel estimator (20) was employed to identify the $L=4$ SIMO CIRs in conjunction with a step size of $\mu_{c}=$ 0.02. The optimal RBF centre set was then computed from the

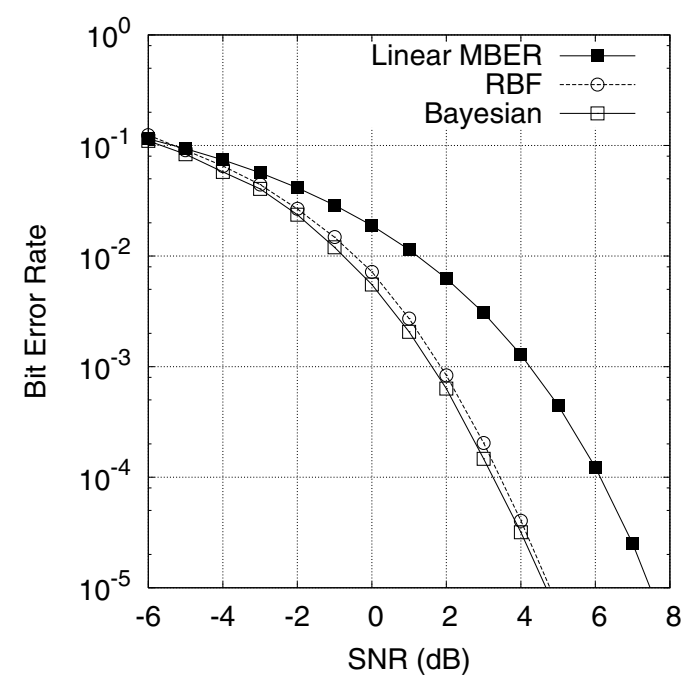

Fig. 3. Bit error rate comparison of three space-time equalisers for the SIMO system listed in Table I. 


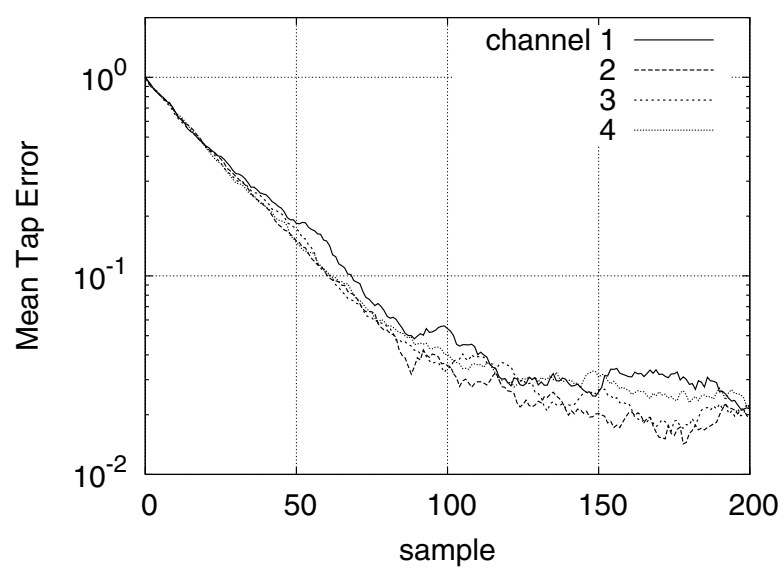

Fig. 4. Learning curve of the LMS SIMO channel estimator averaged over 10 runs for the SIMO system listed in Table $\mathrm{I}$, given $\mathrm{SNR}=1 \mathrm{~dB}$ and the step size $\mu_{c}=0.02$.

estimated SIMO channel matrix. The RBF centres' variance was set to $\sigma_{n}^{2}$. Given $\mathrm{SNR}=1 \mathrm{~dB}$, Fig. 4 characterizes the convergence behaviour of the LMS SIMO channel estimator averaged over 10 runs, in terms of the mean tap error (MTE) expressed as:

$$
\operatorname{MTE}_{l}(k)=\left\|\mathbf{c}_{l}-\hat{\mathbf{c}}_{l}(k)\right\|^{2}, 1 \leq l \leq 4 .
$$

The BER of the SRBF STE is plotted in Fig. 3, in comparison to the two benchmarkers. For the same conditions as those used in Fig. 4, Fig. 5 shows the BER performance of the SRBF STE as a function of the RBF centres' variance. It can be seen from Fig. 5 that the SRBF STE is capable of approaching the Bayesian performance for a large range of RBF variance values.

\section{CONCLUSIONS}

A nonlinear space-time equaliser has been proposed for SIMO systems based on a novel symmetric RBF network. By exploiting the inherent symmetry of the underlying optimal Bayesian STE solution, the proposed SRBF based STE is capable of

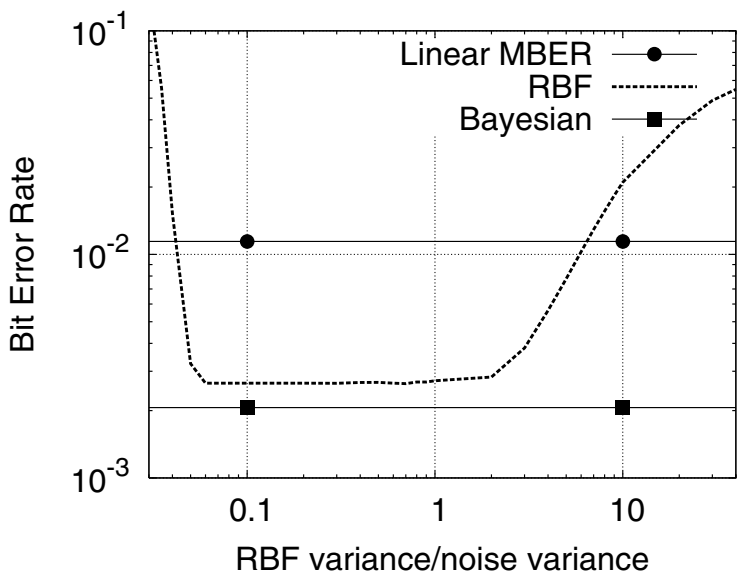

Fig. 5. Bit error rate performance of the SRBF STE as a function of the RBF variance for the SIMO system listed in Table I, given $S N R=1 \mathrm{~dB}$. achieving the optimal Bayesian equalizer's performance. An adaptive implementation of this SRBF based STE has been adopted by estimating the SIMO channels using the LMS channel estimator and computing the optimal RBF centres from the resultant SIMO channel matrix estimate. Our simulation results demonstrated that the performance of this SRBF based STE is robust with respect to the choice of the RBF centres' variance value.

\section{ACKNOWLEDGEMENT}

The financial support of the EPSRC, UK and that of the EU is gratefully acknowledged.

\section{REFERENCES}

[1] G.J. Foschini, "Layered space-time architecture for wireless communication in a fading environment when using multiple antennas," Bell Labs Tech. J., vol.1, no.2, pp.41-59, 1996.

[2] A.J. Paulraj and C.B. Papadias, "Space-time processing for wireless communications," IEEE Signal Processing Magazine, vol.14, no.6, pp.49-83, 1997

[3] R. Kohno, "Spatial and temporal communication theory using adaptive antenna array," IEEE Personal Communications, vol.5, no.1, pp.28-35, 1998.

[4] J.H. Winters, "Smart antennas for wireless systems," IEEE Personal Communications, vol.5, no.1, pp.23-27, 1998.

[5] A.J. Paulraj, D.A. Gore, R.U. Nabar and H. Bolcskei, "An overview of MIMO communications - A key to gigabit wireless," Proc. IEEE, vol.92, no.2, pp.198-218, 2004.

[6] L. Hanzo, M. Münster, B.J. Choi and T. Keller: OFDM and MC-CDMA for Broadband Multi-user Communications, WLANs and Broadcasting, John Wiley - IEEE Press, July 2003, 980 pages.

[7] L. Hanzo, L-L. Yang, E-L. Kuan and K. Yen: Single- and Multi-Carrier DS-CDMA: Multi-User Detection, Space-Time Spreading, Synchronisation, Standards and Networking, IEEE Press - John Wiley, August 2003, 1060 pages.

[8] U. Trautwein, G. Sommerkorn and R.S. Thomä, "A simulation study on space-time equalization for mobile broadband communication in an industrial indoor environment," in Proc. VTC 1999-Spring (Houston, USA), May 16-20, 1999, Vol.1, pp.511-515.

[9] U. Trautwein, D. Hampicke, G. Sommerkorn and R.S. Thomä, "Performance of space-time processing for ISI- and CCI-suppression in industrial scenarios," in Proc. VTC 2000-Spring (Tokyo, Japan), May 15-18, 2000, Vol.3, pp.1894-1898.

[10] P. Stoica, H. Vikalo and B. Hassibi, "Joint maximum-likelihood channel estimation and signal detection for SIMO channels," in Proc. ICASSP'03 (Hong Kong, China), April 6-10, 2003, Vol.IV, pp.13-16.

[11] X. Zhu and R.D. Murch, "Layered space-time equalization for wireless MIMO systems," IEEE Trans. Wireless Communications, vol.2, no.6, pp.1189-1203, 2003.

[12] S. Chen, A. Livingstone and L. Hanzo, "Minimum bite-error rate design for space-time equalization-based multiuser detection," IEEE Trans. Communications, vol.54, no.5, pp.824-832, 2006.

[13] K. Abend and B.D. Fritchman, "Statistical detection for communication channels with intersymbol interference," Proc. IEEE, vol.58, no.5, pp.779-785, 1970.

[14] S. Chen and B. Mulgrew, "Overcoming co-channel interference using an adaptive radial basis function equaliser," Signal Processing, vol.28, no.1, pp.91-107, 1992.

[15] S. Chen, B. Mulgrew and P.M. Grant, "A clustering technique for digital communications channel equalisation using radial basis function networks," IEEE Trans. Neural Networks, vol.4, no.4, pp.570-579, 1993.

[16] S. Chen, S. McLaughlin, B. Mulgrew and P.M. Grant, "Adaptive Bayesian decision feedback equaliser for dispersive mobile radio channels," IEEE Trans. Communications, vol.43, no.5, pp.1937-1946, 1995.

[17] S. Chen, A. Wolfgang, Y. Shi and L. Hanzo, "Space-time decision feedback equalisation using a minimum bit error rate design for singleinput multi-output channels," submitted to IEE Proc. Communications, 2005.

[18] R.O. Duda and P.E. Hart, Pattern Classification and Scene Analysis. New York: Wiley, 1973 